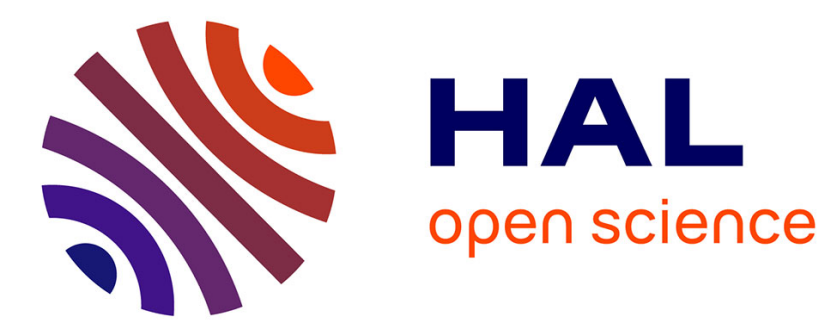

\title{
Strategies for interconnection networks: Some methods from graph theory
}

Jean-Claude Bermond, Charles Delorme, Jean-Jacques Quisquater

\section{To cite this version:}

Jean-Claude Bermond, Charles Delorme, Jean-Jacques Quisquater. Strategies for interconnection networks: Some methods from graph theory. Journal of Parallel and Distributed Computing, 1986, 3 (4), pp.433-449. 10.1016/0743-7315(86)90008-0 . hal-02453396

\section{HAL Id: hal-02453396 https://hal.inria.fr/hal-02453396}

Submitted on 23 Jan 2020

HAL is a multi-disciplinary open access archive for the deposit and dissemination of scientific research documents, whether they are published or not. The documents may come from teaching and research institutions in France or abroad, or from public or private research centers.
L'archive ouverte pluridisciplinaire HAL, est destinée au dépôt et à la diffusion de documents scientifiques de niveau recherche, publiés ou non, émanant des établissements d'enseignement et de recherche français ou étrangers, des laboratoires publics ou privés. 


\title{
Strategies for Interconnection Networks: Some Methods from Graph Theory
}

\author{
J.-C. BERMOND AND C. Delorme \\ Université de Paris-Sud, Centre d'Orsay, Laboratoire de Recherche en Informatique, UA \\ 410 du C.N.R.S., Bât. 490, F-91405 Orsay, France
}

AND

\section{J.-J. QUISQUATER}

Philips Research Laboratory Brussels, Avenue Van Becelaere, 2; B-1170 Brussels, Belgium

\begin{abstract}
Interconnection networks require dense graphs in the sense that many nodes with relatively few links may be connected with relatively short paths. Some recent constructions of such dense graphs with a given maximal degree $\Delta$ and diameter $D$ (known as $(\Delta, D)$ graphs) are reviewed here. The paper also contains an updated table of the best known $(\Delta, D)$ graphs. (C) 1986 Academic Press, Inc.
\end{abstract}

\section{INTRODUCTION}

In this paper we are concerned with the topology of networks. These networks can be distributed computer systems, interconnected memories, telecommunication networks, and so forth. Recent advances in technology, especially the advent of very large scale integrated (VLSI) circuit technology, have enabled very complex interconnection networks to be constructed.

It is well known that telecommunication networks or interconnection networks can be modeled by graphs. Thus, it is of great interest to study the topologies of interconnection networks and their associated graphical properties. A survey of interconnection networks can be found in Feng [31], Uhr [53], or Wu and Feng [57].

Here, we focus on two constraints inherent in networks: the network must be designed so that transmission delay is as small as possible, and each node can be connected by links to only a few other nodes.

The aim of this paper is to describe some of the recent good graphs 


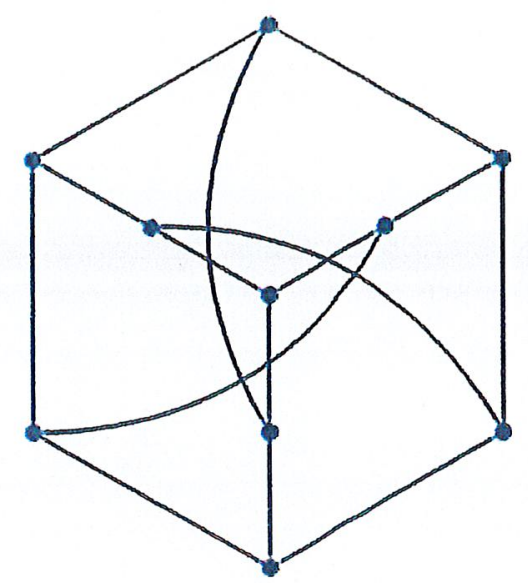

Fí. 1. Petersen graph as drawn by Elspas [29].

achieving these goals. That will be mainly done by examples and figures in order that the constructions can be understood by nonspecialists in graph theory.

\section{Definitions and Notations}

We represent the nodes (respectively links) of interconnection networks by the vertices (resp. edges) of an undirected graph $G$. We use standard terminology (see, for example, Berge [4]). We recall below some important definitions.

The degree of a vertex $x$ is the number of edges incident with $x$. We denote by $\Delta$ the maximum degree of a graph. The distance between two vertices $x$ and $y$ is the length of a shortest path between $x$ and $y$. The diameter is the maximum distance over all the pairs of vertices: it is denoted by $D$. The diameter represents the worst message delay in the network. $\mathrm{A}(\Delta, D)$ graph is a graph with maximum degree $\Delta$ and diameter at most $D$. Note that in the literature the diameter is also noted $k$ and the degree $d$, hence the name $(d, k)$ graphs.

\section{2. $(\Delta, D)$ Graphs and the Moore Bound}

The problem set in the Introduction can be translated in the following graph-theoretic terms. How many vertices can a $(\Delta, D)$ graph have?

If we count the number of vertices which are at distance $0,1,2, \ldots, D$ from a given vertex, we find that there are at most $1+\Delta+\Delta(\Delta-1)+$ $\Delta(\Delta-1)^{2}+\cdots+\Delta(\Delta-1)^{D-1}$ vertices, that is,

$$
\text { if } \Delta=2 \text {, at most } 2 D+1 \text { vertices }
$$

and

$$
\text { if } \Delta \geq 3 \text {, at most } \frac{\Delta(\Delta-1)^{D}-2}{\Delta-2} \text { vertices. }
$$


The preceding bound is due to E. F. Moore (circa 1958) and the graphs achieving this bound are called Moore graphs.

If $D=1$, Moore graphs are the complete graphs (see Fig. 4g of Feng [31]). If $\Delta=2$, Moore graphs are the cycles (or rings) of length $2 D+1$ (see Fig. $4 b$ of Feng [31]). Otherwise, methods of higher algebra show that there can exist Moore graphs only for the following three cases: $D=2$ and $\Delta=3,7$, or 57 (see Biggs [13] for a proof).

The first case with $D=2$ and $\Delta=3$ has 10 vertices and yields the well-known Petersen graph (shown in Fig. 1). In Fig. 2a, the Petersen graph is shown, with the tree issuing from a given vertex and the edges between the last level (points at distance 2 from this vertex). The reader can convince himself that there are always 3 vertices at distance 1 and 6 vertices at distance 2 from any vertex: therefore the Petersen graph is of diameter 2. As we will see in this paper, this graph is very important and in fact is at the origin of most of the known constructions.

For $D=2$ and $\Delta=7$, there exists a graph due to Hoffman and Singleton [40] with 50 vertices attaining the bound. For the Petersen graph it was not too difficult to find the edges to complete the tree (they form a cycle of length 6). Here it might be more convenient to see it as in Fig. 2b, where the reader can check degree 7 and diameter 2 .

For $D=2$ and $\Delta=57$, there might exist a Moore graph with 3250 vertices, but no one has been able to construct (or prove the nonexistence of) such a graph.

In view of the preceding results, it is interesting to find graphs that have a great number of vertices, not too far from the Moore bound. This problem was first posed by Elspas [29].
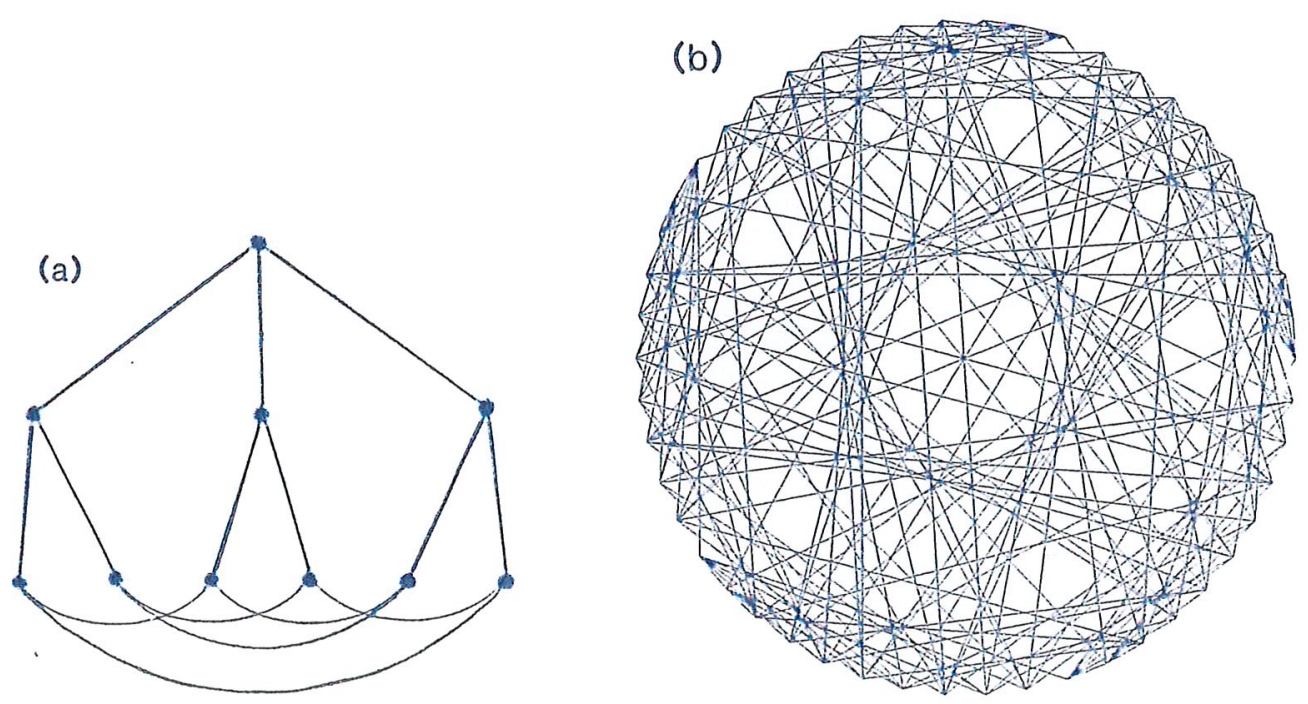

FIG. 2. Moore graphs. (a) Petersen graph. (b) Hoffman and Singleton graph. 


\section{Generalized Chordal Rings}

Figure 3a shows the Petersen graph drawn in a different manner. One can see that the vertices are labeled with the integers modulo 10, and that the vertex $i$ is joined to vertices $i+4, i+5, i+6$ if $i$ is even and to vertices $i+2, i+5, i+8$ if $i$ is odd (all the numbers are to be taken modulo 10). That is the first example of what is called a generalized chordal ring, the definition being:

The graph $G$ is a generalized chordal ring if we can label the vertices with integers modulo $n$ and find a divisor $q$ of $n$ such that vertex $i$ is joined to vertex $j$ if and only if vertex $i+q$ is joined to vertex $j+q$.

For the Petersen graph we have $n=10$ and $q=2$. If all the edges of the form $(i, i+1)$ appear in the graph, we say that it is a chordal ring; indeed such a graph can be viewed as obtained from a ring by adding chords in a regular manner.

Figure $3 b$ shows the Petersen graph again, with another labeling: the vertex
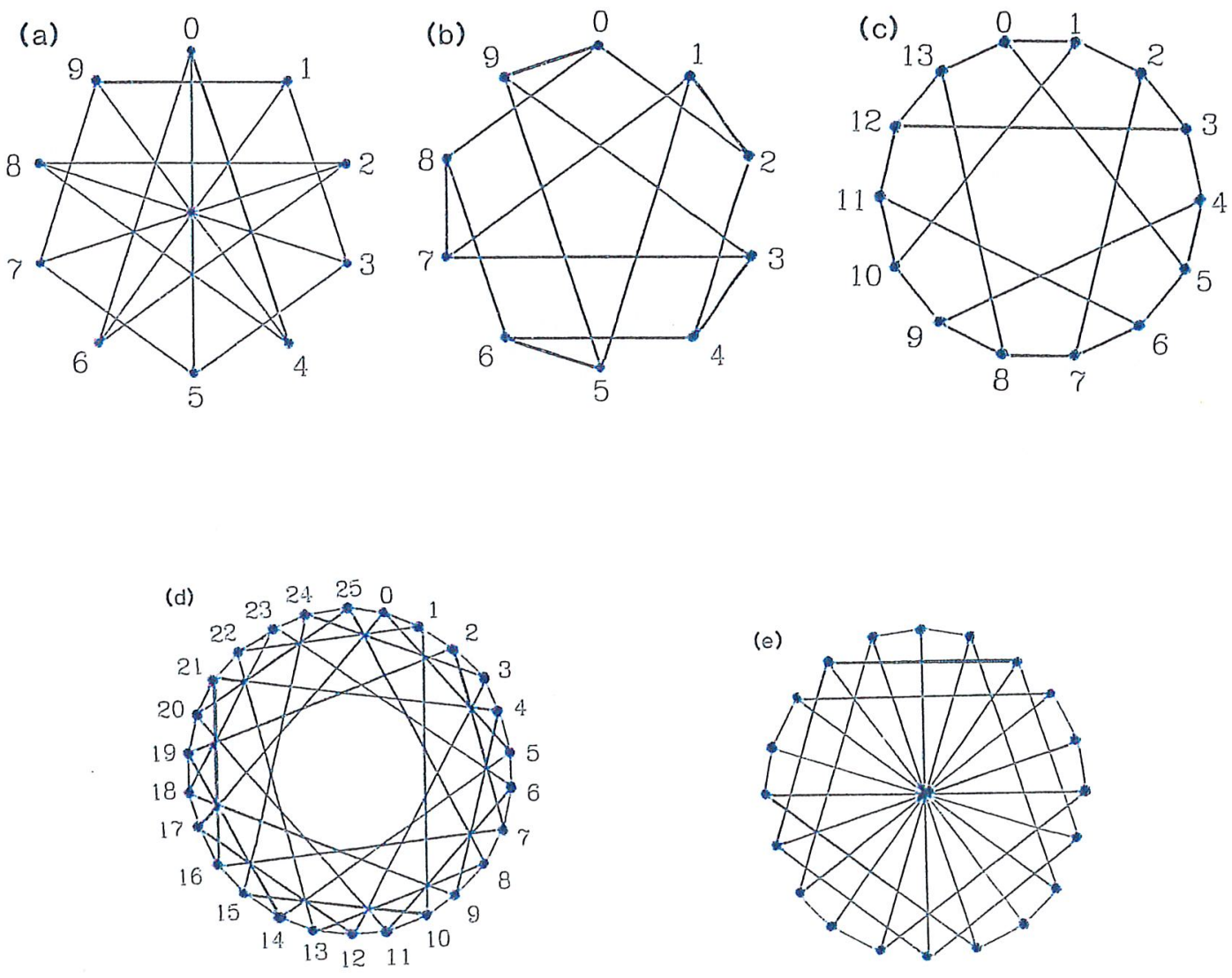

FIG. 3. Generalized chordal rings. (a) Petersen graph. (b) Petersen graph again. (c) Heawood graph. (d) Chordal graph on 26 vertices. (e) An optimal $(3,3)$ graph. 
$i$ is joined to vertices $i+2, i+8, i+9$ if $i$ is even and the vertex $i$ is joined to $i+1, i+4, i+6$ if $i$ is odd.

Figure $3 \mathrm{c}$ shows a chordal ring on 14 vertices, of degree 3 and diameter 3 (known as the Heawood graph). Here $q=2$ and vertex $i$ is joined to vertices $i+1, i+5, i+13$ if $i$ is even and vertex $i$ is joined to vertices $i+1$, $i+9, i+13$ if $i$ is odd. Figure $3 d$ shows a chordal graph on 26 vertices, with degree 4 and diameter 3 (here $q=2$ ).

The two graphs of Figs: $3 c$ and $d$ belong to a general family of graphs associated to finite geometries (see Delorme [22]). Finally Fig. 3e shows a chordal graph on 20 vertices, with degree 3, diameter 3 (here $q=4$ ). In fact this graph has the maximum possible number of vertices among the $(3,3)$ graphs (indeed the Moore bound, which cannot be attained, is 22 and parity arguments rule out the value 21 ).

The construction of these graphs using computers has been done by different authors, for example, Arden and Lee [3] and Doty [26, 28], who obtained some of the largest known $(\Delta, D)$ graphs. These graphs are denoted in Table II by $C R$ (Chordal Ring). For example, there exists a chordal ring on 708 vertices, of degree 3 and diameter 10. (See Table II in the Conclusion.)

\section{GRAPHS ON AlPHABETS}

One way to construct good graphs is to label the vertices with words on a given alphabet, with a rule relating two different words. This presentation has a double advantage as it gives easy ways of construction and easy methods for routing messages.

The first example is again the Petersen graph: the vertices are labeled with words of length 5 from the alphabet $\{0,1\}$ (or binary sequences), containing exactly three ones, where two vertices are joined if they have exactly one digit in common. For example, 01110 is joined to 11001 because they have only a 1 in common, in second position (see Fig. 4a). Using words of length 7 with four ones, this method gives the graph of Fig. $4 \mathrm{~b}$ due to Akers [1]. These graphs are known in the literature as odd graphs; their parameters satisfy

$$
\Delta=D+1, n=\left(\begin{array}{c}
2 \Delta+1 \\
\Delta
\end{array}\right) \text {. }
$$

Other important graphs of this kind are de Bruijn graphs [16], frequently rediscovered (see Ralston [49] for historical references beginning in 1894). The vertices are labeled with words of length $D \geq 2$, on an alphabet of $\delta \geq 2$ letters. Two words are connected if the last $D-1$ letters of one are the same as the first $D-1$ letters of the other (see Fig. $4 c$, with $D=3$, and $\Delta=3$ ). The graph has $\delta^{D}$ vertices. If $D \geq 3$ and $\delta \geq 3$, its diameter is $D$ and its maximum degree is $2 \delta$. A description of these graphs exist also in terms of Kronecker products (see Quisquater [48]).

The Kautz graphs [44] are deduced from de Bruijn graphs in an easy manner: delete words where there are two consecutive identical letters (see Fig. 4c). 

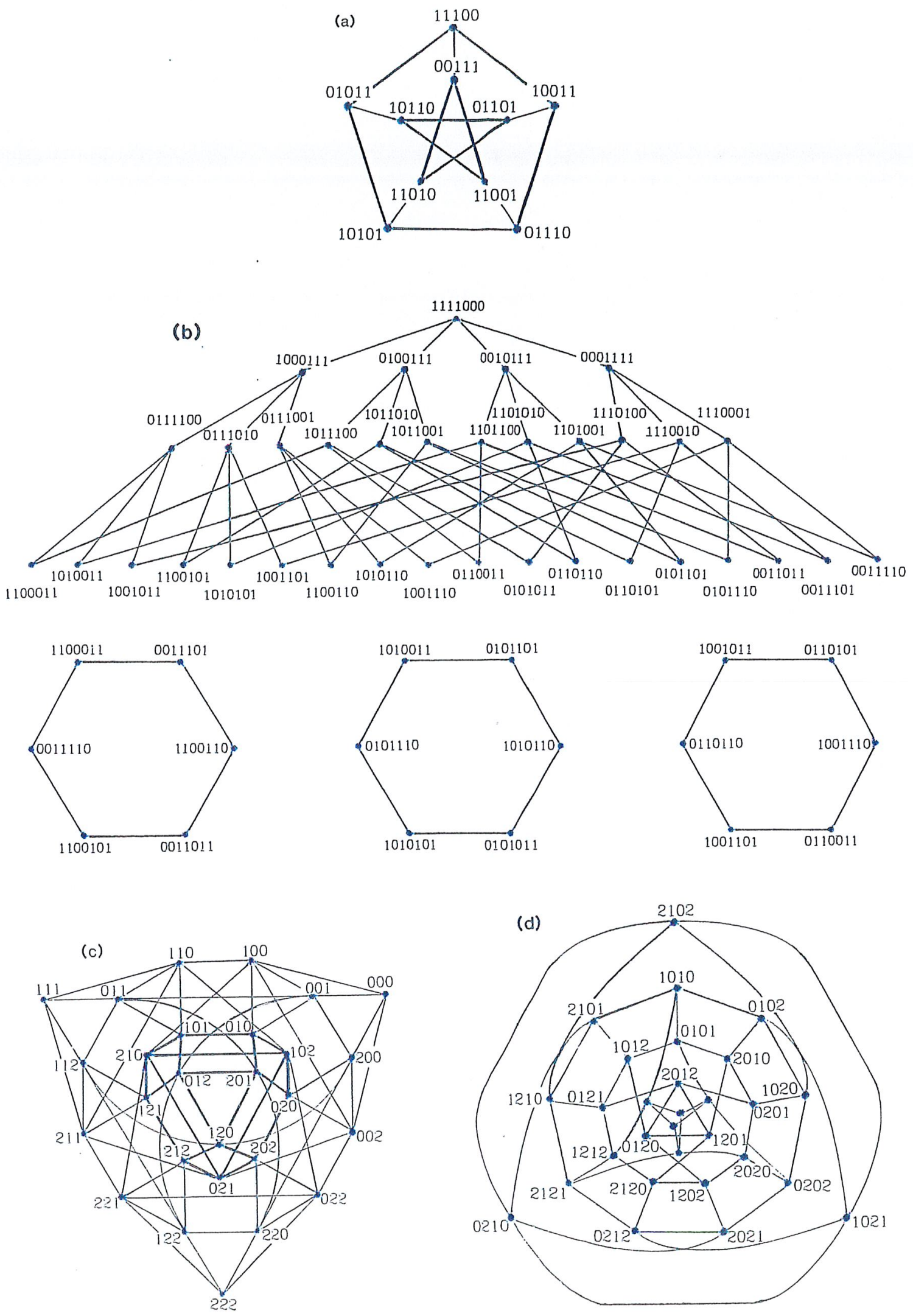

FIG. 4. Graphs on alphabets. (a) Petersen graph. (b) Akers graph. (c) Kautz graph from de Bruijn graph. (d) Modified Kautz graph. 
If $D \geq 3$ and $\delta \geq 3$, Kautz graphs have maximum degree $2 \delta-2$, diameter $D$, with $\delta(\delta-1)^{D-1}$ vertices. Kautz graphs have been rediscovered by Fiol, Alegre, and Yebra [33, 35] (these authors give two other interesting descriptions of these graphs), Imase and Itoh [42], and Reddy, Kuhl, Hosseini, and Lee [50].

De Bruijn graphs and Kautz graphs are not regular. Thus, we can try to add vertices without modifying maximum degree and diameter. Indeed, Bond [14] was able to add $2 \delta-1$ vertices to Kautz graphs (see Fig. 4c).

These graphs have been generalized by Delorme and Farhi [24]; Imase and Itoh [41, 42]; and Gomez, Fiol, Yebra, and Fabrega [36, 39]. For example, the construction of [42] allows graphs with orders $n$ that are not powers of $\delta$. The vertices are the integers modulo $n$, the vertex $i$ being joined to the vertices $-\delta i-t$, where $t=1,2, \ldots, \delta$.

\section{PRODUCTS}

Let us draw the Petersen graph again (Fig. 5a), as the union of two pentagons joined in a particular manner. More exactly the vertex $(1, \alpha)$ of the external cycle is joined to the vertex $(2,2 \alpha)$ of the internal cycle, where $\alpha$
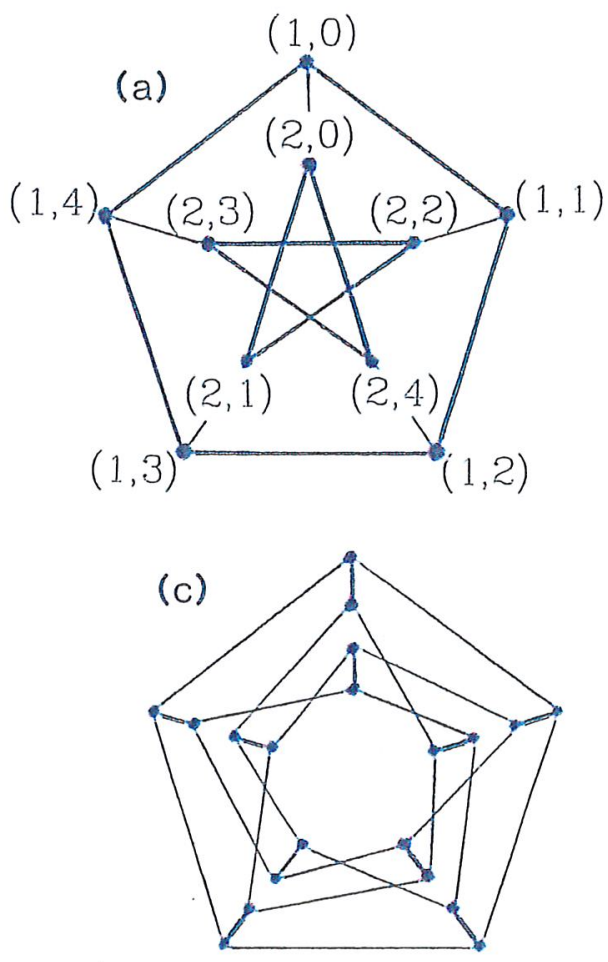

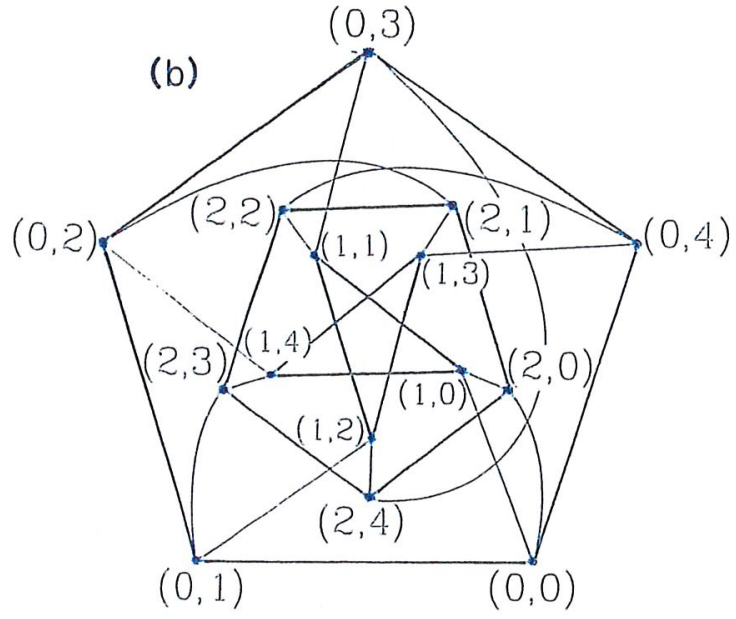

(d)

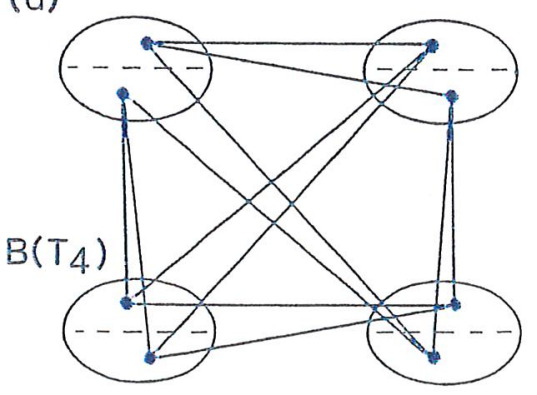

FiG. 5. Products. (a) Petersen graph. (b) An optimal $(4,2)$ graph. (c) An optimal $(3,3)$ graph. (d) Using bipartite graphs. 
is an integer modulo 5 . As an example, $(1,3)$ is joined to $(2,1)$. In fact the graph can be viewed as a special product of an edge and a pentagon. This product is defined in full generality in Bermond, Delorme, and Farhi $[8,9]$. here we give only two more examples which are in fact optimal graphs.

The first one is the product of a triangle and a pentagon (Fig. 5b). It has 15 vertices and is of degree 4 and diameter 2 (it has been shown by Erdös, Fajtlowicz, and Hoffman [30] that such a graph cannot have 16 vertices). In this graph the vertex $(i, \alpha)$ is joined to $(i+1,2 \alpha)$ where $i$ is an integer modulo 3 and $\alpha$ an integer modulo 5 .

The second example (Fig. 5c) is the product of a pentagon and a graph of 4 vertices consisting of two disjoint edges. The 5 isomorphic graphs on 4 vertices are shown with heavy lines. Products of cycles have been also considered by von Conta [20].

Other examples can be found in Bermond, Delorme, and Farhi [8, 9]; some of them give largest known $(\Delta, D)$ graphs (see Table II). For example, the optimal $(5,2)$ graph on 24 vertices is also of this kind.

A new simple product using bipartite graphs has been described recently by Gómez and Fiol [38]. It is shown in Fig. 5d. The ellipses represent copies of a bipartite graph $B$ of order $n$, even diameter $D$ and maximum degree $\triangle$ and with the two stable parts (separated by a dashed line) of same order. The resulting graph $B\left(\mathbb{T}_{4}\right)$ is obtained by adding perfect matchings between some of these parts, according to the figure. It has $4 n$ vertices, diameter $D$ and degree $\Delta+3$. Other generalizations which give good values in the table have been discovered recently by Gómez [37].

\section{COMPOUND GRAPHS}

Here we try again to draw copies of a given graph and to join the copies, but now we want to have only a few edges between two copies.

Figure 6a shows this idea with 6 copies of pentagons and where there exists exactly one edge between any pair of copies. The degree is now 3 and the diameter is at most 5: an upper bound on the diameter is given by the sum of diameters of any pair of pentagons plus one for the edge joining the two copies. The reader can check that its diameter is in fact 5. This graph is denoted by $C_{5}\left[K_{6}\right]$ : it is the compound of pentagons into a complete graph on 6 vertices.

In Fig. $6 \mathrm{~b}$ we have 5 copies of graphs isomorphic to a bistar (drawn in heavy lines) connected to each other by exactly two edges. This graph now has degree exactly 3 , diameter 4 , and 30 vertices. This compound has been used by different authors (Uhr [52] and Bermond, Delorme, and Quisquater [10]). In particular, in the case where there is the same number of edges between two copies, that gives rise to some of the largest $(\Delta, D)$ graphs. For example, a graph on 2550 vertices, of diameter 5 and degree 8 , is obtained by taking 51 copies of a Hoffman-Singleton graph: it was the largest known $(8,5)$ graph for some time. 

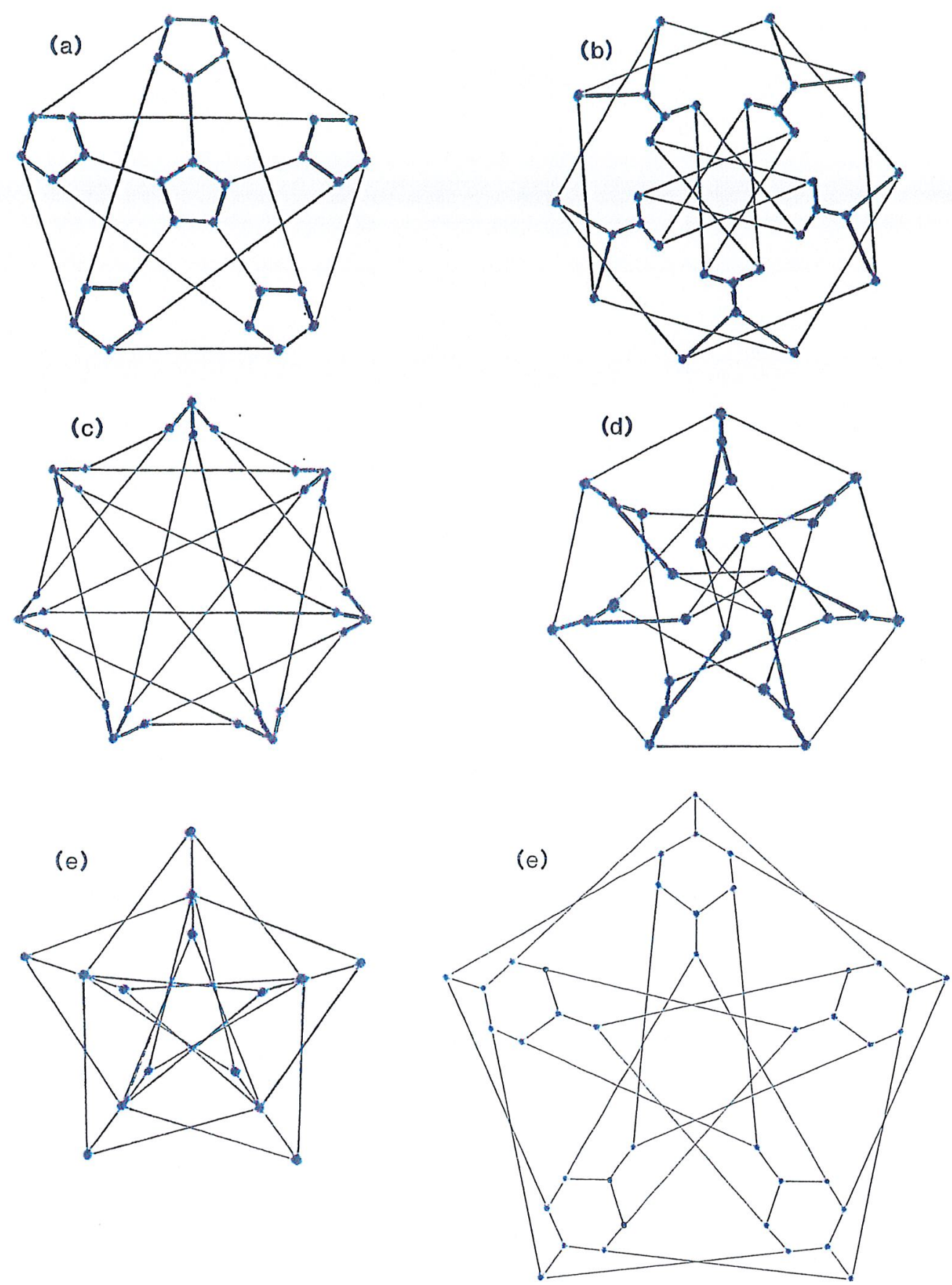

FIG. 6. Compound graphs. (a) Compound of pentagons into a complete graph on six vertices. (b) Five copies of graphs isomorphic to a bistar. (c, d) Graphs obtained by compounding seven copies of a star; the graph in (d) is known as the Coxeter graph. (e) A bipartite graph (left) and a resulting compound graph (right).

The diameter of the compound graph depends on the choice of the edges between the copies. For example, in Figs. $6 \mathrm{c}$ and d we show two graphs obtained by compounding 7 copies of a star (there is exactly one edge between each pair of copies). While the two graphs obtained have 28 vertices and 
degree 3, the first one, in Fig. 6c, has diameter 5 and the second one, in Fig. $6 \mathrm{~d}$, diameter 4 (the latter graph is known as the Coxeter graph).

As the diameter is dependent on the choice of the edges connecting the copies and as the calculation of the diameter is not easy, it is useful to have short calculations and this requires construction of highly symmetrical graphs. Part of the symmetry can be seen in the figures: Fig. $6 \mathrm{~b}$ is invariant under a rotation of $2 \pi / 5$ and Fig. $6 d$ under a rotation of $2 \pi / 7$.

Some of these ideas have been used by Quisquater [48] and Alegre, Fiol, and Yebra [2] for constructing some good graphs (for example, the graph with 70 vertices, of degree 3 and diameter 5). Jerrum and Skyum [43] have compounded trees or other graphs into de Bruijn graphs, obtaining the largest known $(\Delta, D)$ graphs for fixed degree and very large diameter. A simpler construction of such graphs in the case $\Delta=3$, using coding theory, has been given by Chung [18].

It is also possible to compound two different kinds of graphs into a bipartite graph. Figure 6e shows a bipartite graph on 15 vertices and the resulting graph in which the 5 vertices of degree 6 belonging to one class of the bipartite graph have been replaced by hexagons. This graph has 40 vertices, degree 3 , and diameter 6 , which follows from the fact that the replacement has been done in a clever manner (an awkward replacement could have given only a diameter 8). Calculations and other examples can be found in Bermond, Delorme, and Quisquater [11]. In fact some particular cases were considered previously in Wegner [54].

It is sometimes possible to improve the number of vertices by taking more carefully into account the structure of the copies and the way of connecting them.

One idea is that between two copies that are not directly connected, there is a possibility of finding short distances from the use of short paths in the other copies. Thus one obtains a graph on 2650 vertices, with degree 8 and diameter 5, by taking 53 copies (instead of 51) of a Hoffman-Singleton graph, owing to the fact that it has a Hamilton path. It is described in Delorme and Quisquater [25].

Another idea is to take more than one and less than $n$ edges between copies of a graph on $n$ vertices. This idea is used in Alegre, Fiol, and Yebra [2], Fiol and Fabrega [34], Gómez [37], and Gómez and Fiol [38].

\section{CONCLUSion}

We hope to have given an idea of the richness of the subject and how different methods can lead to interesting graphs. In fact there exist many other methods or constructions, too complicated to be explained here, for example, the methods using geometric objecis as projective planes, generalized ngons, polarities, and so forth (see Delorme [22]). Some large graphs have 
been constructed by Carlsson, Cruthirds, Sexton, and Wright [17], using some group theory, which is quite relevant for the search of highly symmetrical graphs. Some constructions also produce asymptotically good graphs and can be found in the referenced papers.

Several tables of the largest known $(\Delta, D)$ graphs have been given in the literature, for example, by Storwick [51] in 1970, and recently by Memmi and Raillard [46], Leland, Finkel, Qiao, Solomon, and Uhr [45], and the current authors [10]. We give here an updated table (see Table II). To show the progress that has been made on the subject and the work that remains to be done, we give here the number of vertices for some peculiar values of $\Delta$ and $D$ from these tables versus the Moore bound.

Other related problems are also of interest; for example, we can restrict our attention to bipartite graphs (see Delorme [23] and Doty [27]). We can also consider the analogous problems when sets of processors share a communication medium such as a bus: this structure is used in the ILLIAC IV, in the computer interconnection system MICRONET (see Wittie and van Tilborg [56]), or in other networks introduced by Finkel and Solomon [32] and Wittie [55]. This problem belongs to the theory of hypergraphs: see Bermond, Bond, and Saclé [7], Bermond, Bond, and Peyrat [6], and Doty [27].

Other parameters have to be considered also, such as the vulnerability of the constructed networks and the routing algorithms. For more details we refer the reader to the surveys of Bermond, Bond, Paoli, and Peyrat [5], Bermond, Homobono, and Peyrat [12], and Chung [19]. Applications in VLSI should be considered: a first step in this direction can be found in Peterson and Ting [47] or in the work of J. Bently, C. Leiserson, and others. Perhaps some of our figures will be helpful in the design of some interconnections in VLSI.

TABLE I

Some Comparisons between Tables of Largest Known $(\Delta, D)$ GraphS

\begin{tabular}{rrrrrrr}
\hline & Storwick [51] & LFQSU [4.5] & BDQ [10] & & & \\
$(\Delta, D)$ & 1970 & 1981 & 1982 & Nov. 1984 & July 1986 & Moore bound \\
\hline$(9,3)$ & 150 & 150 & 585 & 585 & 585 & 658 \\
$(10,5)$ & 910 & 5,550 & 7,550 & 7,650 & 10,000 & 73,811 \\
$(3,10)$ & 216 & 525 & 536 & 708 & 708 & 3,070 \\
$(6,10)$ & 9,465 & 59,049 & 131,232 & 180,180 & 188,370 & $14,648,437$ \\
$(10,10)$ & 170,685 & $9,765,625$ & $14,981,200$ & $46,249,515$ & $47,059,200$ & $4,358,480,501$ \\
\hline
\end{tabular}

\section{APPENDIX: NOTATIONS AND REFERENCES}

$\otimes B \quad$ Kronecker product of bipartite graphs defined in Delorme [23].

$B(G) \quad$ Product of a bipartite graph $B$ by an oriented graph $G$ (Gómez and Fiol [38]).

$B_{1} \nabla_{i} B_{2} \quad$ Product of bipartite graphs (Gómez and Fiol [38]).

$B_{1} \Sigma_{i} B_{2} \quad$ Another product of bipartite graphs (Gómez and Fiol [38]).

$B W \quad$ Construction of Brouwer and Wilbrink based on finite geometries [15]. 
TABLE II

LARGEST KNOWN $(\Delta, D)$ GRAPHS (SePtember 1986) ${ }^{a, b}$

\begin{tabular}{|c|c|c|c|c|c|c|c|c|c|}
\hline$\Delta^{D}$ & 2 & 3 & 4 & 5 & 6 & 7 & 8 & 9 & 10 \\
\hline 3 & (10) & $C_{s} * F_{4}$ & $\begin{array}{r}Y F A \\
38\end{array}$ & $\begin{array}{r}Y F A \\
70\end{array}$ & $\begin{array}{l}\mathrm{H}_{2} r \\
128\end{array}$ & $\begin{array}{l}C R \\
180\end{array}$ & $\begin{array}{r}Y F A \\
286\end{array}$ & $\begin{array}{l}C R \\
462\end{array}$ & $\begin{array}{r}C R \\
708\end{array}$ \\
\hline 4 & $K_{s} * C_{s}$ & $\begin{array}{r}P * F_{4} \\
40\end{array}$ & $\begin{array}{r}C_{s} * C_{19} \\
95\end{array}$ & $\begin{array}{l}H_{s}^{\prime} \\
364\end{array}$ & $\begin{array}{l}H_{s} r \\
731\end{array}$ & $\begin{array}{r}C R \\
856\end{array}$ & $\begin{array}{r}C R \\
1,872\end{array}$ & $\begin{array}{r}G C C \\
4,352\end{array}$ & $\begin{array}{r}G C C \\
13,056\end{array}$ \\
\hline 5 & $K_{s} * x_{s}$ & $\begin{array}{r}L \\
70\end{array}$ & $\begin{array}{l}Q_{4} r \\
174\end{array}$ & $\begin{array}{r}H_{s}^{\prime} d \\
532\end{array}$ & $\begin{array}{r}H_{4} r \\
2,734\end{array}$ & $\begin{array}{l}H_{4} i d r \\
2,988\end{array}$ & $\begin{array}{r}C R \\
7,600\end{array}$ & $\begin{array}{r}G C C \\
15,360\end{array}$ & $\begin{array}{r}G C C \\
52,224\end{array}$ \\
\hline 6 & 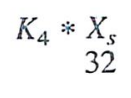 & $\begin{array}{r}C_{5} * C_{21} \\
105\end{array}$ & $\begin{array}{r}P * m(32) \\
320\end{array}$ & $\begin{array}{r}G C C \\
992\end{array}$ & $\begin{array}{r}H_{5} r \\
7,817\end{array}$ & $\begin{array}{r}G C C \\
13,056\end{array}$ & $\begin{array}{r}G C C \\
32,256\end{array}$ & $\begin{array}{r}P_{4}^{\prime} \Lambda_{p} H_{4} \\
\quad 72,345\end{array}$ & $\begin{array}{r}L \Lambda_{p} H_{4} \\
199,290\end{array}$ \\
\hline 7 & $\frac{H S}{50}$ & $\begin{array}{l}C R \\
122\end{array}$ & $\begin{array}{r}(15) * m(32) \\
480\end{array}$ & $\begin{array}{r}C R \\
1,550\end{array}$ & $\begin{array}{r}H_{4} r<H_{5} \\
10,546\end{array}$ & $\begin{array}{l}H_{5} \wedge K_{1} \\
35,154\end{array}$ & $\begin{array}{r}K_{4} \Lambda_{p} H_{5} \\
93,744\end{array}$ & $\begin{array}{r}(24) \Lambda_{p} H_{5} \\
304,668\end{array}$ & $\begin{array}{r}Q_{5} \nabla_{1} H_{5} \\
1,218,672\end{array}$ \\
\hline 8 & $\begin{array}{l}P_{7}^{\prime} \\
57\end{array}$ & $\begin{array}{r}H S \approx F_{4} \\
200\end{array}$ & $\begin{array}{l}Q_{7} r \\
807\end{array}$ & $\begin{array}{r}K_{1,1} \Sigma_{7} Q_{5} \\
2,808\end{array}$ & $\begin{array}{r}H_{7} r \\
39,223\end{array}$ & $\begin{array}{r}K_{1,1} \Sigma_{7} H_{5} \\
70,308\end{array}$ & $\begin{array}{r}K_{5,5} \Sigma_{16} H_{5} \\
234,360\end{array}$ & $\begin{array}{l}P_{4} \Sigma_{7} H_{5} \\
820,260\end{array}$ & $\begin{array}{r}Q_{4} \Sigma_{5} H_{5} \\
3,984,120\end{array}$ \\
\hline
\end{tabular}




\begin{tabular}{|c|c|c|c|c|c|c|c|c|c|}
\hline 9 & $\begin{array}{r}P_{8}^{\prime} d \\
74\end{array}$ & $\begin{array}{r}Q_{6}^{\prime} \\
585\end{array}$ & $\begin{array}{l}Q_{5}\left(T_{4}\right) \\
1,248\end{array}$ & $\begin{array}{r}H S] C_{103}[ \\
5.150\end{array}$ & $\begin{array}{r}H_{8} r \\
74,906\end{array}$ & $\begin{array}{l}H_{7} \wedge K_{1} \\
215,688\end{array}$ & $\begin{array}{l}K_{4} \Lambda_{p} H_{7} \\
666,672\end{array}$ & $\begin{array}{r}H S \Lambda_{p} H_{7} \\
3,019,632\end{array}$ & $\begin{array}{r}Q_{7} \nabla_{1} H_{7} \\
15,686,400\end{array}$ \\
\hline 10 & $\begin{array}{l}P_{9}^{\prime} \\
91\end{array}$ & $\begin{array}{l}Q_{8}^{\prime} d \\
650\end{array}$ & $\begin{array}{r}\left(Q_{s}\left\{T_{s}\right\}\right)^{\prime} B W \\
1,755\end{array}$ & $\begin{array}{r}K_{1} \Sigma_{8} Q_{7} \\
10,000\end{array}$ & $\begin{array}{r}H_{9} r \\
132,869\end{array}$ & $\begin{array}{r}H_{8} \wedge K_{1} \\
486,837\end{array}$ & $\begin{array}{l}K_{5,5} \Delta_{6} H_{7} \\
1,764,720\end{array}$ & $\begin{array}{r}H S \Lambda_{p} H_{9} \\
7,714,494\end{array}$ & $\begin{array}{r}Q_{7} \Sigma_{2} H_{7} \\
47,059,200\end{array}$ \\
\hline 11 & $\begin{array}{r}P_{9}^{\prime} d \\
94\end{array}$ & $Q_{8}^{\prime} d$ & $\begin{array}{l}Q_{7}\left(T_{4}\right) \\
3,200\end{array}$ & $\begin{array}{r}K_{1} \Sigma_{8} Q_{8} \\
14,625\end{array}$ & $\begin{array}{r}H_{7}\left(T_{4}\right) \\
156,864\end{array}$ & $\begin{array}{r}K_{1}, 1 \Sigma_{7} H_{6} \\
898,776\end{array}$ & $\begin{array}{l}K_{6,6} \Sigma_{{ }_{6}} H_{6} \\
4,044,492\end{array}$ & $\begin{array}{r}P_{7} \Sigma_{7} H_{8} \\
21,345,930\end{array}$ & $\begin{array}{r}Q_{7} \Sigma_{6} H_{8} \\
179,755,200\end{array}$ \\
\hline 12 & $\begin{array}{l}P_{11}^{\prime} \\
133\end{array}$ & $\frac{Q_{8}^{\prime} d}{780}$ & $\begin{array}{r}Q_{8}^{\prime} * X_{8} \\
4,680\end{array}$ & $\begin{array}{r}K_{1,1} \Sigma_{7} Q_{9} \\
21,320\end{array}$ & $\begin{array}{r}H_{11} r \\
354,323\end{array}$ & $\begin{array}{l}K_{1,1} \Sigma_{7} H_{9} \\
1,727,180\end{array}$ & $\begin{array}{l}K_{7,7} \Sigma_{8} H_{9} \\
8,370,180\end{array}$ & $\begin{array}{r}P_{6} \Sigma_{7} H_{9} \\
48,493,900\end{array}$ & $\begin{array}{r}Q_{8} \Sigma_{6} H_{9} \\
466,338,600\end{array}$ \\
\hline 13 & $\begin{array}{l}P_{11}^{\prime} d \\
136\end{array}$ & $\begin{array}{l}Q_{8}^{\prime} d \\
845\end{array}$ & $\begin{array}{l}Q_{9}\left(T_{4}\right) \\
6,560\end{array}$ & $\begin{array}{r}Q_{6}^{\prime} * m\left(P_{7}^{\prime}\right) \\
33,345\end{array}$ & $\begin{array}{r}H_{9}\left(T_{4}\right) \\
531,4,40\end{array}$ & $\begin{array}{r}H_{11} \wedge K_{1} \\
2,657,340\end{array}$ & $\begin{array}{l}K_{8,8} \Sigma_{6} H_{8} d \\
10,257,408\end{array}$ & $\begin{array}{r}P_{9} \Sigma_{1} H_{9} \\
72,541,560\end{array}$ & $\begin{array}{r}Q_{9} \Sigma_{8} H_{9} \\
762,616,400\end{array}$ \\
\hline 14 & $\begin{array}{l}P_{13}^{\prime} \\
183\end{array}$ & $\begin{array}{l}Q_{8}^{\prime} d \\
910\end{array}$ & $\begin{array}{l}Q_{9}\left(T_{3}\right) \\
8,200\end{array}$ & $K_{1} \sum_{51,240} Q_{11}$ & $\begin{array}{r}H_{13} r \\
804,481\end{array}$ & $\begin{array}{r}K_{1} \Sigma_{8} H_{11} \\
6,200,460\end{array}$ & $\begin{array}{l}K_{7.7} \Sigma_{8} H_{11} \\
29,762,208\end{array}$ & $\begin{array}{r}P_{9} \Sigma_{7} H_{11} \\
164,755,080\end{array}$ & $\begin{array}{r}Q_{8} \Sigma_{88} H_{11} \\
1,865,452,680\end{array}$ \\
\hline 15 & $\begin{array}{r}P_{13}^{\prime} d \\
186\end{array}$ & $\begin{array}{r}\left(\otimes Q_{2,4}\right)^{\prime} \\
1,215\end{array}$ & $\begin{array}{l}Q_{11}\left(T_{4}\right) \\
11,712\end{array}$ & $\begin{array}{r}K_{1} \Sigma_{8} Q_{11} \\
58,560\end{array}$ & $\begin{array}{r}H_{11}\left(T_{4}\right) \\
1,417,248\end{array}$ & $\begin{array}{r}K_{1} \Sigma_{8} H_{11} \\
7,086,240\end{array}$ & $\begin{array}{r}K_{6,6} \Sigma_{16} H_{11} d \\
35,947,392\end{array}$ & $\begin{array}{r}P_{11} \Sigma_{1} H_{11} \\
282,740,976\end{array}$ & $\begin{array}{r}Q_{11} \Sigma_{6} H_{11} \\
3,630,989,376\end{array}$ \\
\hline 16 & $\begin{array}{r}P_{13}^{\prime} d \\
197\end{array}$ & $\begin{array}{r}\left(\otimes Q_{5}\right)^{\prime} \\
1,600\end{array}$ & $\begin{array}{l}Q_{11}\left(T_{5}\right) \\
14,640\end{array}$ & $\begin{array}{r}\left(\otimes H_{5}\right)^{\prime} \\
132,496\end{array}$ & $\begin{array}{r}H_{11}\left(T_{5}\right) \\
1,771,560\end{array}$ & $\begin{array}{r}K_{1} \Sigma_{6} H_{15} \\
14,882,658\end{array}$ & $\begin{array}{l}K_{9,9} \Sigma_{66} H_{15} \\
86,882,544\end{array}$ & $\begin{array}{r}P_{9} \Sigma_{7} H_{11} \\
585,652,704\end{array}$ & $\begin{array}{r}Q_{11} d \Sigma_{66} H_{15} \\
7,394,669,856\end{array}$ \\
\hline
\end{tabular}

${ }^{a}$ Circled values are optimal.

${ }^{b}$ See the Appendix for notations and references for the table. 
$C_{n} \quad$ Cycle with $n$ vertices.

CR Chordal ring (Arden and Lee [3] and Doty [26, 28]).

$d \quad$ Operation such as doubling some edges, described in Delorme $[22$, Sects. 7, 8, 9].

$F_{4} \quad$ Graph from Galois Field on 4 elements (Bermond, Delorme, and Farhi [8]).

$G<B \quad$ Compound of a graph $G$ in a bipartite graph $B$ (Gómez and Fiol [38]).

$G_{1} \Lambda G_{2} \quad$ Product defined in Delorme and Quisquater [25].

$G_{1} * G_{2}$ Product of graphs $G_{1}$ and $G_{2}$ defined in Bermond, Delorme, and Farhi $[8,9]$.

$G_{1} \Lambda_{p} B_{2} \quad$ Product defined in Gómez [37].

$G^{\prime} \quad$ Factor graph of some $G$ by a polarity (Delorme [22]).

$\left.G_{1}\right] G_{2}[$ Compound defined in Delorme and Quisquater [25].

$G_{1}\left\{G_{2}\right\} \quad$ Product defined in Delorme and Quisquater [25].

GCC Generalized cube-connected cycles defined in Carlsson, Cruthirds, Sexton, and Wright [17].

$H_{q} \quad$ Generalized hexagon (Delorme [22]).

HS Hoffman and Singleton graph [40].

$i \quad$ Addition of vertices on some edges (in Delorme [22, Sect. 9]).

$K_{n} \quad$ Complete graph on $n$ vertices.

$L \quad$ Graph on alphabet found by C. Lente (July 1986).

$m(G) \quad$ Half-part of a given graph (Delorme [21]).

$P \quad$ Petersen graph.

$P_{q} \quad$ Incidence graph associated to a projective plane (Delorme [22]).

$Q_{q} \quad$ Generalized quadrangle (Delorme [22]).

$Q_{u, v} \quad$ Generalized quadrangle (Delorme [23]).

$r \quad$ Replacement of a vertex by some complete graph (Quisquater [48]).

$T_{i} \quad$ Tournament on $i$ vertices.

$X_{8} \quad$ Graph on 8 vertices described in Bermond, Delorme, and Farhi [9].

YFA Compound graph from the construction of Alegre, Fiol, and Yebra [2].

\section{ACKNOWLEDGMENTS}

Thanks are due to J. Bond, M. Davio, P. Delsarte, C. Peyrat and the referees for helpful comments.

\section{REFERENCES}

1. Akers, S. On the construction of $(d, k)$ graphs. IEEE Trans. Electron Comput. EC-14 (1965), 488.

2. Alegre, I., Fiol, M. A., and Yebra, J. L. A. Some large graphs with given degree and diameter. J. Graph Theory 10 (1986), 219-224.

3. Arden, B. W., and Lee, H. A regular network for multicomputer systems. IEEE Trans. Comput. C-31 (1982), 60-69. 
4. Berge, C. Graphs and Hypergraphs. North-Holland, New York, 1973

5. Bermond, J. -C., Bond, J., Paoli, M., and Peyrat, C. Graphs and interconnection networks: Diameter and vulnerability. In Surveys in Combinatorics, Invited Papers for the Ninth British Combinatorial Conf. London Math. Soc. Lecture Note Series, Vol 82. Cambridge Univ. Press, London/New York, 1983, pp. 1-30.

6. Bermond, J. -C., Bond, J., and Peyrat, C. Bus interconnection networks with each station on two buses. In Proc. Coll. Int. Parallel Algorithms and Architectures, Marseille, Apr. 1986. North-Holland, New York, in press.

7. Bermond, J. -C., Bond, J., and Saclé, J. -F. Large hypergraphs of diameter 1. In Graph Theory and Combinatorics, Proc. Cambridge Combinatorial Conference. Academic Press, Orlando, Fla., 1984, pp. 19-28.

8. Bermond, J. -C., Delorme, C., and Farhi, G. Large graphs with given degree and diameter, II. J. Combin. Theory Ser. B 36 (1984), 32-48.

9. Bermond, J. -C., Delorme, C., and Farhi, G. Large graphs with given degree and diameter, III Proc. Coll. Cambridge, 1981; Ann. Discrete Math. 13 (1982), 23-32.

10. Bermond, J. -C., Delorme, C., and Quisquater, J. -J. Tables of large graphs with given degree and diameter. Inform. Process. Lett. 15 (1982), 10-13. [A regularly updated version may be obtained from the authors.]

11. Bermond, J. -C., Delorme, C., and Quisquater, J. -J. Grands graphes de degré et diamètre fixés. Ann. Discrete Math. 17 (1983), 65-73.

12. Bermond, J. -C., Homobono, N., and Peyrat, C. Large fault-tolerant interconnection networks. Proc. First Japan Conference on Graph Theory and Applications, Hakone, Japan, June 1986.

13. Biggs, N. Algebraic Graph Theory. Cambridge Tracts in Math. No. 67. Cambridge Univ. Press, London, 1974.

14. Bond, J. Construction de grands graphes d'interconnexions. Thesis, LRI, Université ParisSud, Orsay, 1984.

15. Brouwer, A. E., and Wilbrink, H. A. Private communication, Jan. 1982.

16. de Bruijn, N. G. A combinatorial problem. Koninkl. Nederl. Acad. Wetensch. Proc. Ser A 49 (1946), 758-764.

17. Carlsson, G. E., Cruthirds, J. E., Sexton, H. B., and Wright, C. G. Interconnection networks based on a generalization of cube-connected cycles. IEEE Trans. Comput. C-34 (1985), 769-772.

18. Chung, F. R. K. Sparse graphs with small diameters. Proc. 3rd International Conference on Combinatorial Mathematics, 1985, Ann. New York Acad. Sci., to appear.

19. Chung, F. R. K. Diameter of communication networks, In Short Course on the Mathematics of Information Processing Amer. Math. Soc., Providence, R. I., 1986. Vol. 34, pp. 1-18.

20. von Conta, C. Torus and other networks as communication networks with up to some hundred points, IEEE Trans. Comput. C-32 (1983), 657-666.

21. Delorme, C. Two notes on large graphs. Rapport de Recherche n 172, LRI, Orsay, 1984.

22. Delorme, C. Grands graphes de degré et diamètre donnés. J. Européen Combin. 6 (1985), 291-302.

23. Delorme, C. Large bipartite graphs with given degree and diameter. J. Graph Theory 9 (1985), 325-334.

24. Delorme, C., and Farhi, G. Large graphs with given degree and diameter, I. IEEE Trans. Comput. C-33 (1984), 857-860.

25. Delorme, C., and Quisquater, J. -J. New large compound graphs. Manuscript, 1984. 
26. Doty, K. W. Large regular interconnection networks. Proc. IEEE Third International Conference on Distributed Computing Systems, Miami, Oct. 1982, pp. 312-317.

27. Doty, K. W. Dense bus connection networks. Proc. IEEE 1983 Int. Conf. on Parallel Processing, pp. 158-160.

28. Doty, K. W. New design for dense processor interconnection networks. IEEE Trans. Comput. C-33 (May 1984), 447-450.

29. Elspas, B. Topological constraints on interconnection-limited logic. Proc. IEEE Fifth Symposium on Switching Circuit Theory and Logical Design, IEEE S-164, 1964, pp. 133-147.

30. Erdös, P., Fajtlowicz, S., and Hoffman, A. J. Maximum degree in graphs of diameter 2. Networks 10 (1980), 87-90.

31. Feng, T. Y. A survey of interconnection networks. Computer 14 (1981), 12-27.

32. Finkel, R. A., and Solomon, M. H. The lens interconnection strategy. IEEE Trans. Comput. C.30 (1981); 960-965.

33. Fiol, M. A., Alegre, I. and Yebra, J. L. A. Line digraph iterations and the $(d, k)$ problem for directed graphs. Proc. Tenth Int. Symposium Comput. Architecture, Stockholm, 1983, pp. 174-177.

34. Fiol, M. A., and Fabrega, J. Algunos grafos compuestas. Stochastica 7, 2 (1983), 137-143.

35. Fiol, M. A., Yebra, J. L. A., and Alegre, I. Line digraph iterations and the $(d, k)$ digraph problem. IEEE Trans. Comput. C-33 (1984), 400-403.

36. Fiol, M. A., Yebra, J. L. A., and Fabrega, J. Sequence graphs and interconnection networks. Proc. Ninth British Combinatorial Conf., Southampton; Ars Combin. 16-A (1985), 7-14.

37. Gómez, J. Diametro y vulnerabilidad en redes de interconexión. Thesis, Universitat Politecnica de Catalunya, Barcelona, Sept. 1986.

38. Gómez, J., and Fiol, M. A. Dense compound graphs. Proc. Tenth British Combinatorial Conf., Glasgow; Ars Combin. 20-A (1985), 211-237.

39. Gómez, J., Fiol, M. A., and Yebra, J. L. A. Graphs on alphabets as models for large computer networks. Submitted for publication.

40. Hoffman, A. J., and Singleton, R. R. On Moore graphs with diameter 2 and 3. IBM J. Res. Develop. 4 (1960), 497-504.

41. Imase, M., and Itoh, M. Design to minimize diameter on building-block network. IEEE Trans. Comput. C-30 (1981), 439-442.

42. Imase, M., and Itoh, M. A design for directed graphs with minimum diameter. IEEE Trans. Computers C-32 (1983), 782-784.

43. Jerrum, M., and Skyum, S. Families of fixed degree graphs for processor interconnection. IEEE Trans. Comput. C-33 (1984), 190-194.

44. Kautz, W. H. Bounds on directed $(d, k)$ graphs. In Theory of Cellular Logic Networks and Machines. AFCRL-68-0668, SRI Project 7258, final report, 1968, pp. 20-28.

45. Leland, W. E., Finkel, R., Qiao, L., Solomon, M. H., and Uhr, L. High density graphs for processor interconnection. Inform. Process. Lett. 12 (1981), 117-120.

46. Memmi, G., and Raillard, Y. Some new results about the $(d, k)$ graph problem. IEEE Trans. Comput. C-31 (1982), 784-791.

47. Peterson, G. L., and Ting, Y. -H. Trade-offs in VLSI for bus communication networks. In 1982-1983 Computer Science and Computer Engineering Research Review. University of Rochester, Rochester, N. Y., pp. 23-32.

48. Quisquater, J. -J. New constructions of large graphs with fixed degree and diameter. To appear. 
49. Ralston, A. de Bruijn sequences. A model example of the interaction of discrete mathematics on the computer science. Math. Mag. 55 (1982), 131-143.

50. Reddy, S. M., Kuhl, J. G., Hosseini, S. H., and Lee, H. On digraphs with minimum diameter and maximum connectivity. Proc. 20th Annual Allerton Conference, Oct. 1982, pp. 1018-1026.

51. Storwick, R. M. Improved construction techniques for $(d, k)$ graphs. IEEE Trans. Comput. C-19 (1970), 1214-1216.

52. Uhr, L. Compounding denser $(d, k)$ graph architectures for computer networks. Comput. Sci. Tech. Rep. N 414, University of Wisconsin, Madison, Jan. 1981.

53. Uhr, L. Algorithm-Structured Computer Arrays and networks. Academic Press, Orlando, Fla., 1984.

54. Wegner, G. Graphs with given diameter and a coloring problem. Unpublished paper, Oct. 1976.

55. Wittie, L. D. Communication structures for large networks of microcomputers. IEEE Trans. Comput. C-30 (1981), 264-273.

56. Wittie, L. D., and van Tilborg, A. M. MICROS, a distributed operating system for MICRONET, a reconfigurable network computer, IEEE Trans. Comput. C-29 (1980), $1133-1144$.

57. Wu, C. L., and Feng, T. Y. Tutorial: Interconnection Networks for Parallel .nd Distributed Processing. IEEE Comput. Soc. New York, 1984. 